\title{
Independent and combined association of parity and short pregnancy with obesity and weight change among Indian women
}

\author{
Shilpi Gupta, Satwanti Kapoor
}

Department of Anthropology, University of Delhi, Delhi, India; ${ }^{*}$ Corresponding Author: satwanti@yahoo.com

Received 31 January 2012; revised 13 February 2012; accepted 6 March 2012

\begin{abstract}
Objective: To investigate association of parity and short pregnancy with obesity and weight change in Aggarwal Baniya women. Method: A cross-sectional analysis was carried out on a representative sample of $\mathbf{3 0 7}$ adult Aggarwal Baniya women aged 30 - 50 years (mean age: $38.7 \pm$ 4.87) using multistage cluster sampling method. Weight, height, various skinfold thicknesses, waist and hip circumference were measured using standardized protocol. Various indices of obesity (BMI, WHR, WHtR, GMT) were calculated subsequently. Comparison groups were defined by the number of births (parity), short pregnancies and total pregnancies. Mean change in weight and other obesity markers were examined for each group separately. Correlation analysis was applied to see the association of childbearing on obesity. Linear regression was applied as an effective measure. Results: There was a gain in weight $(3.16 \mathrm{~kg})$ and increase in other obesity markers (BMI: $1.29 \mathrm{~kg} / \mathrm{m}^{2}$; WC: $2.38 \mathrm{~cm}$; HC: $3.83 \mathrm{~cm}$ ) with each increase in each parity. Significant and positive correlation $(p<$ 0.001) was found between obesity and parity. However, negative association was found between short pregnancy and obesity parameters (BMI: $-0.767 \mathrm{~kg} / \mathrm{m}^{2}$ ). Conclusion: Among other risk factors, high parity number may be associated with obesity in women. Therefore, interventions to prevent obesity should be targeted at women prior to initiation of childbearing. However, the impact of reproductive wastage in the form of short pregnancies on women's obesity needs further exploration.
\end{abstract}

Keywords: Women; Parity; Short Pregnancy; Weight Gain; Central Obesity

\section{INTRODUCTION}

Obesity as a health problem needs no introduction in the present day context. The task of the day is to identify the risk factors for obesity. Reproductive factors, especially parity, have been found positively associated with weight gain and the onset of obesity in population-based studies [1-4]. In these studies, excess weight gain associated with a single birth has been estimated to be $2-3 \mathrm{~kg}$ $[5,6]$. It has been reported that parity affects body fat and BMI [7] and, in a cohort of older women, Bastian et al. [8] found that higher rates of obesity were associated with an increasing number of children regardless of SES and other confounding factors. Women's weight tends to increase the most during their first pregnancy compared with subsequent pregnancies [9,10]. Smith et al. [5] found that subgroups of women tend to be more vulnerable to weight gain with pregnancy and to risk of becoming overweight.

Grand multiparity is not only considered as a major risk factor for many obstetric and gynecological disorders and maternal deaths, but also for the development of obesity. The extent of this relationship and the pathways leading to the parity-associated weight gain in the different populations are, however, yet to be understood. Even less is known about changes in measures of central adiposity associated with childbearing.

Short pregnancies influence changes in weight and girth. Maternal fat deposition begins early in pregnancy and has been estimated at $1.5 \mathrm{~kg}$ by 7 weeks gestation [11]. Therefore, early fat gain may be retained long term even after a short pregnancy. However, previous studies have not evaluated this major confounder. To the best of our knowledge only one study has reported association of short pregnancy with weight change [12].

The influence of obesity on blood pressure was demonstrated in a socio-economically stratified sample by Gupta and Kapoor [13], pointing towards the risk of developing a high load of obesity among the socioeconomically advanced urban Indians thus making them vul- 
nerable to obesity related health risks. Women are also more burdened than men with the undesirable sociopsychological effects of obesity, because the society as a whole favors thinness, especially for women $[14,15]$.

The prevalence of high obesity among Aggarwal Baniya females has been documented [13]. Although effect of various risk factors on obesity has been studied but the effect of reproductive and maternal health on obesity has received less attention. Based on evidence that pregnancy may adversely affect body weight and central adiposity, we explored these relationships further among Aggarwal Baniya women.

\section{MATERIALS AND METHODS}

\subsection{Target Population}

The present study was conducted on Aggarwal Baniya women of Delhi, India. Data for the present cross-sectional study was collected by household survey on 307 adult Aggarwal Baniyas women aged 30 - 50 years (mean age: $38.7 \pm 4.87$ years). All women reported themselves to be vegetarian with majority of them were Housewives/home makers $(94.8 \%)$. Non-response rate for all the variables of interest was $7.2 \%$ in all cases. Final analysis was performed on the basis of 285 women with complete information. Information was collected during household visits using a proforma that included information on anthropometry and reproductive performance.

\subsection{Data Collection}

Participants were interviewed through structured proforma. Anthropometric measurements including height, weight, skinfold thickness, waist and hip circumference were obtained using standardized procedures.

Body weight was measured by using spring balance to the nearest $500 \mathrm{gm}$, stature with the help of Martin's Anthropometer to the nearest $\mathrm{mm}$. Waist circumference and hip circumference were measured with a non stretchable flexible steel tape to the nearest mm. Body mass index was calculated as weight divided by height squared $\left(\mathrm{kg} / \mathrm{m}^{2}\right)$, and categorized as normal $(\leq 25.0)$, overweight ( $\geq 25.0$ but $\leq 30.0$ ), and obese $(\geq 30.0)$ [16]. Abdominal obesity was defined as waist circumference $\geq 80$ centimetres in women [17]. Waist-to-hip ratio was calculated by dividing waist circumference by hip circumference. High waist hip ratio was defined as $\geq 0.85$ in women [17]. Grand mean thickness (GMT) was calculated by adding all skinfold thicknesses taken at different sites divided by number of skinfold sites. Waist to height ratio was calculated as the ratio of waist circumference $(\mathrm{cm})$ to height (cm). High WHtR was defined as $\geq 0.50$ [18].

Measurements were conducted by trained personnel and all instruments were calibrated once weekly. The pro- tocol for the study was reviewed and approved by Ethical Committee of the Department of Anthropology, Faculty of Sciences, University of Delhi, India.

\subsection{Statistical Analysis}

Parity and short pregnancy were classified as 1 through $3+$ based on the self-reported number of live births and short pregnancies. Short pregnancies included abortions, miscarriages and stillbirths. Few women reported parity and short pregnancy of +3 ; therefore, women with parity and short pregnancy of +3 were clubbed with parity and short pregnancy of three respectively. Women with 1 - 2 live birth and 1 short pregnancy were used as the reference group for parity and short pregnancy respectively. Total pregnancy was defined as combine of live births and short pregnancies. It was classified as 1 through more than 5 . Women with more than 5 pregnancies were clubbed together and recoded as group 4.

Data was analysed using Statistical Package for Social Science (SPSS), version 15.0. The results are given as means and standard deviations (SD). ANOVA test was used to compare the measurements between groups. Pearson's correlation coefficient was used to evaluate the correlations between parity, short pregnancy, total pregnancy and the obesity markers. The question of how parity, short pregnancy and total pregnancy is associated with obesity markers was examined using multiple regression analysis, adjusting for age.

\section{RESULTS}

Mean weight, height, BMI, waist circumference, waist/ hip ratios, waist/height ratio and grand mean thickness are shown by parity, short pregnancy and total pregnancy groups in Table 1 (Figure 1). The mean value of weight, BMI and other regional obesity indices signifycantly increased with increase in parity and total pregnancy. However, with the increase in number of miscarriage, the mean value of weight, BMI and GMT decreased though regional obesity indices didn't show any significant association. The number of women with BMI $>30 \mathrm{~kg} / \mathrm{m}^{2}$ was higher in high parity groups.

Table 2 shows association of obesity markers with parity, short pregnancy and total pregnancy. There were significant positive correlations between parity number, total pregnancy and various obesity markers. However, negative correlations were found between short pregnancies and obesity markers.

Table 3 shows regression coefficients $(\beta)$ of parity, short pregnancy and total pregnancy on various obesity markers. There was a significant increase in various obesity markers with the increase in parity and total pregnancy number. However with each increased short pregnancy, there was significant decrease in the values of 
Table 1. Change in obesity markers according to categories of parity, short pregnancies and total pregnancies.

\begin{tabular}{|c|c|c|c|c|c|c|c|c|c|c|}
\hline \multicolumn{2}{|c|}{ Obesity parameters } & Age & Weight & BMI & $\begin{array}{c}\text { BMI }(\%) \\
>30 \mathrm{~kg} / \mathrm{m}^{2}\end{array}$ & WHR & WHtR & WC & $\mathrm{HC}$ & GMT \\
\hline \multirow{5}{*}{$\begin{array}{c}\text { Parity } \\
\mathrm{N}=285\end{array}$} & $\begin{array}{c}\text { group } 1 \\
(1-2)\end{array}$ & $37.7 \pm 4.54$ & $65.6 \pm 11.44$ & $28.6 \pm 4.44$ & 36.1 & $0.86 \pm 0.07$ & $0.60 \pm 0.07$ & $90.3 \pm 10.42$ & $104.5 \pm 10.1$ & $30.9 \pm 6.53$ \\
\hline & group $2(=3)$ & $38.6 \pm 3.46$ & $70.1 \pm 11.72$ & $30.1 \pm 5.30$ & 47.4 & $0.86 \pm 0.08$ & $0.61 \pm 0.07$ & $93.2 \pm 10.73$ & $108.7 \pm 10.7$ & $32.6 \pm 6.66$ \\
\hline & group $3(>3)$ & $44.2 \pm 7.79$ & $75.0 \pm 8.57$ & $32.2 \pm 3.68$ & 55.6 & $0.83 \pm 0.07$ & $0.63 \pm 0.07$ & $96.4 \pm 10.40$ & $116.5 \pm 9.99$ & $32.9 \pm 9.36$ \\
\hline & $\mathrm{F}$ & 23.39 & 10.14 & 8.11 & & 2.91 & 3.29 & 4.88 & 16.6 & 1.79 \\
\hline & $\mathrm{p}$ & $<0.001$ & $<0.001$ & $<0.001$ & & $<0.056$ & $<0.039$ & $<0.008$ & $<0.001$ & NS \\
\hline \multirow{4}{*}{$\begin{array}{c}\text { Short } \\
\text { pregnancy } \\
\mathrm{N}=162\end{array}$} & $\begin{array}{l}\text { group1 } \\
(=1)\end{array}$ & $37.9 \pm 4.69$ & $71.3 \pm 11.18$ & $30.8 \pm 5.22$ & 51.9 & $0.85 \pm 0.07$ & $0.61 \pm 0.06$ & $93.1 \pm 9.30$ & $109.6 \pm 11.6$ & $34.6 \pm 6.41$ \\
\hline & group $2(=2)$ & $40.1 \pm 3.16$ & $67.7 \pm 16.20$ & $29.5 \pm 6.36$ & 50.0 & $0.85 \pm 0.06$ & $0.61 \pm 0.08$ & $91.7 \pm 13.8$ & $107.4 \pm 12.8$ & $31.2 \pm 8.80$ \\
\hline & group $3(\geq 3)$ & $41.6 \pm 7.13$ & $65.6 \pm 11.48$ & $28.6 \pm 4.45$ & 22.0 & $0.90 \pm 0.09$ & $0.62 \pm 0.06$ & $94.3 \pm 9.43$ & $104.7 \pm 9.74$ & $27.5 \pm 4.49$ \\
\hline & $\mathrm{p}$ & $<0.001$ & $<0.05$ & $<0.10$ & & $<0.001$ & NS & NS & NS & $<0.001$ \\
\hline \multirow{6}{*}{$\begin{array}{c}\text { Total } \\
\text { pregnancy } \\
\mathrm{N}=285\end{array}$} & group $1(=2)$ & $36.0 \pm 3.73$ & $64.9 \pm 9.77$ & $27.6 \pm 3.48$ & 21.5 & $0.84 \pm 0.07$ & $0.57 \pm 0.06$ & $87.0 \pm 9.95$ & $103.6 \pm 9.32$ & $30.1 \pm 5.01$ \\
\hline & group $2(=3)$ & $38.2 \pm 4.34$ & $70.9 \pm 10.43$ & $30.9 \pm 4.26$ & 3.8 & $0.88 \pm 0.06$ & $0.63 \pm 0.06$ & $95.5 \pm 9.67$ & $109.0 \pm 9.35$ & $34.0 \pm 5.71$ \\
\hline & group $3(=4)$ & $39.3 \pm 4.26$ & $98.9 \pm 12.68$ & $30.0 \pm 5.87$ & 13.2 & $0.84 \pm 0.07$ & $0.60 \pm 0.06$ & $90.6 \pm 8.61$ & $108.4 \pm 12.5$ & $32.7 \pm 8.34$ \\
\hline & group $4(\geq 5)$ & $41.0 \pm 5.79$ & $68.0 \pm 12.95$ & $29.4 \pm 5.01$ & 20.8 & $0.87 \pm 0.09$ & $0.62 \pm 0.08$ & $94.1 \pm 12.26$ & $107.8 \pm 11.8$ & $30.0 \pm 7.27$ \\
\hline & $\mathrm{F}$ & 13.97 & 3.40 & 6.11 & & 6.18 & 11.7 & 9.72 & 3.49 & 5.65 \\
\hline & $\mathrm{p}$ & $<0.001$ & $<0.01$ & $<0.001$ & & $<0.001$ & $<0.001$ & $<0.001$ & $<0.01$ & $<0.001$ \\
\hline
\end{tabular}

Table 2. Correlations of obesity markers with parity, short pregnancy and total pregnancy.

\begin{tabular}{|c|c|c|c|c|c|c|}
\hline \multirow{2}{*}{ Obesity parameters } & \multicolumn{2}{|c|}{ Parity } & \multicolumn{2}{|c|}{ Short pregnancy } & \multicolumn{2}{|c|}{ Total pregnancy } \\
\hline & $\mathrm{r}$ & $\mathrm{p}$ value & $\mathrm{r}$ & $\mathrm{p}$ value & $\mathrm{r}$ & $P$ value \\
\hline Body weight (Kg) & $0.223^{* *}$ & $<0.001$ & $-0.220^{* *}$ & $<0.005$ & 0.040 & NS \\
\hline BMI & $0.219^{* *}$ & $<0.001$ & $-0.182^{*}$ & $<0.021$ & 0.080 & NS \\
\hline Waist circumference & $0.184^{* *}$ & $<0.002$ & 0.063 & NS & $0.191^{* *}$ & $<0.001$ \\
\hline WHR & -0.087 & NS & $0.344^{* *}$ & $<0.001$ & $0.189^{* *}$ & $<0.001$ \\
\hline WHtR & $0.168^{* *}$ & $<0.005$ & 0.109 & NS & $0.219^{* *}$ & $<0.001$ \\
\hline GMT & 0.081 & NS & $-0.373^{* *}$ & $<0.001$ & $-0.126^{*}$ & $<0.046$ \\
\hline
\end{tabular}

${ }^{*} \mathrm{p}<0.01 ;{ }^{* *} \mathrm{p}<0.05$.

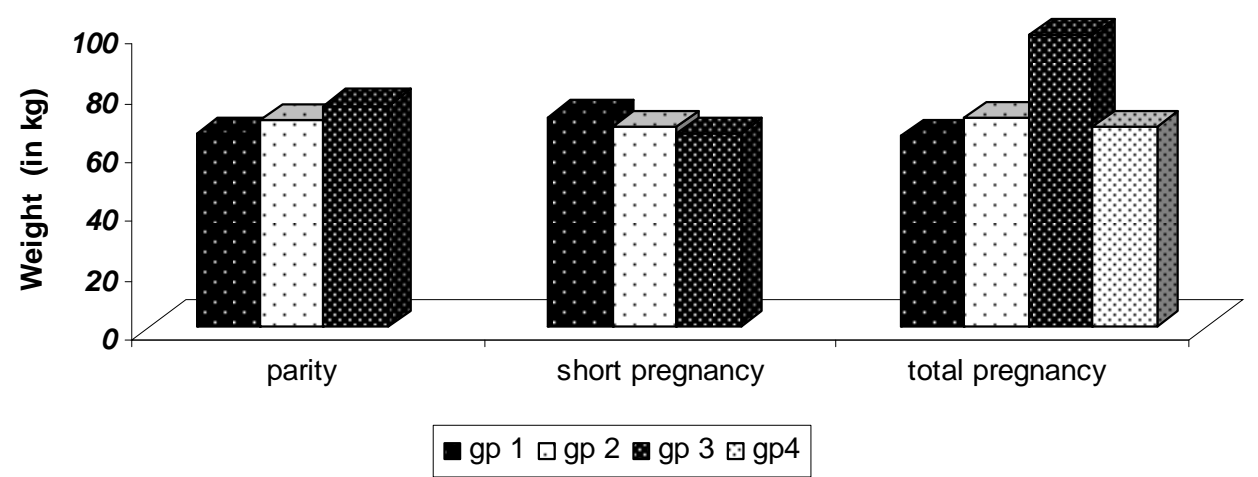

Figure 1. Difference in mean weight change in various groups of parity, short and total pregnancy. 
Table 3. Multiple Linear Regression for parity, short pregnancy and total pregnancy on various obesity markers.

\begin{tabular}{lcccccccccc}
\hline \multirow{2}{*}{ Obesity parameters } & \multicolumn{3}{c}{ Parity } & \multicolumn{3}{c}{ Short pregnancy } & \multicolumn{3}{c}{ Total pregnancy } \\
\cline { 2 - 10 } & $\beta$ & SE & p & B & SE & p & $\beta$ & SE & p \\
\hline Weight & $\mathbf{3 . 1 6}$ & 0.82 & $<0.001$ & $-\mathbf{2 . 2 1 6}$ & 0.777 & $<0.005$ & 0.296 & 0.444 & NS \\
BMI & $\mathbf{1 . 2 9}$ & 0.34 & $<0.001$ & $-\mathbf{0 . 7 6 7}$ & 0.328 & $<0.021$ & 0.248 & 0.184 & NS \\
WC & $\mathbf{2 . 3 8}$ & 0.76 & $<0.002$ & 0.524 & 0.657 & NS & $\mathbf{1 . 3 0 7}$ & 0.399 & $<0.001$ \\
Hip circumference & $\mathbf{3 . 8 3}$ & 0.758 & $<0.001$ & $-\mathbf{1 . 8 7 7}$ & 0.699 & $<0.008$ & 0.554 & 0.416 & NS \\
WHR & -0.01 & 0.005 & NS & $\mathbf{0 . 0 2 0}$ & 0.004 & $<0.001$ & $\mathbf{0 . 0 0 9}$ & 0.003 & $<0.001$ \\
WHtR & $\mathbf{0 . 0 1}$ & 0.005 & $<0.005$ & 0.006 & 0.004 & NS & $\mathbf{0 . 0 1 0}$ & 0.003 & $<0.001$ \\
GMT & 0.70 & 0.54 & NS & $-\mathbf{2 . 0 1 5}$ & 0.422 & $<0.001$ & $-\mathbf{0 . 5 7 3}$ & 0.285 & $<0.04$ \\
\hline
\end{tabular}

$\beta$-Regression coefficient; SE-standard error; p-significance level.

various obesity markers.

\section{DISCUSSION}

It was found that values of weight, BMI and other obesity markers were higher in grand multiparous (group $2 \& 3$ ) and multigravid women (group 2, $3 \& 4$ ) than in non-grand multiparous and multigravid women (group 1 $\&$ 2). There was a statistically significant correlation between parity number, total pregnancy with various obesity indices. This study generally supports the existing literature that there are positive relationships between parity and weight $[9,10,19]$. However, in the present study an inverse relation between number of short pregnancies and obesity markers has been found. There was a decrease in various obesity indices with the increase in number of miscarriage.

It has been previously demonstrated that obesity is common among Aggarwal Baniya women [13]. The cause of the high prevalence of obesity in women is still unclear. Mechanisms have been proposed to explain this association of number of children and obesity among women, with reference to excess deposit of fat tissue that accumulates, preferentially in the femoral area during pregnancy [20]. Kapoor et al. [21] also mentioned the importance of trunkal fat in pregnant women which may act as a shock absorbing pad in protecting the growing fetus besides as the source of energy needed during this period. Behavioral factors may also contribute to gains in both weight and central adiposity associated with pregnancy or its aftermath. For example, heritable predisposition to gain weight, changes in lifestyle during and after pregnancy (smoking cessation, dietary intake, and physical activity) as well as cultural perceptions of women's body image, employment and maternal roles have been reported to have an impact on postpartum weight gain or retention [22-24].

In the present study, increase in regional obesity indices with child bearing indicates greater deposition of fat in abdominal area. This present finding is supported by another study which reported that 68 percent of gestational fat gain is deposited in the trunk [25] and excess gain remaining at $1 \mathrm{y}$ postpartum tends to be located in the trunk [26]. Continued excess waist circumference gain after first and higher order births may be related to physical changes in body structure such as expansion of the abdominal muscles, ribcage, and uterus during the third trimester of pregnancy to accommodate fetal growth as well as greater adipose tissue deposition. This may explain that cumulative excess gains in waist circumference are due to changes in central adiposity.

A study has reported $7 \%$ increase in risk of obesity for each additional child, adjusting for age, race, household income, work status, physical activity, tobacco use and alcohol use [27]. In a cohort of older women, Bastian et al. [8] found that higher rates of obesity were associated with an increasing number of children regardless of SES and other confounding factors. A positive correlation of waist-hip ratio (WHR) with parity has been reported in many cross-sectional studies [2,20,28]. These findings provide important prospective evidence that childbirth contributes to the development of obesity. Childbirth (either primiparous or multiparous) appeared to increase a woman's risk of developing obesity relatively soon after delivery. This finding is troubling because most women who become obese subsequent to childbirth remain overweight or obese in the years following the perinatal period.

Number of studies has reported that parity-related weight gain or retention has the greatest impact on obesity risk with the first childbirth; the contribution of subsequent births to obesity appears to be nominal $[6,29,30]$. However present study differ from these studies and therefore further research is needed by examining whether the interpregnancy intervals of multiparous women contribute to this phenomenon or not.

Short pregnancies influence changes in weight and 
girth, however, previous studies have not evaluated this major confounder. To our knowledge, this is the first cross-sectional study to see the association of various obesity markers with number of short pregnancies. Only one study in literature has examined the association of short pregnancies with weight and waist circumference [12]. However, our results are contradictory to their finding. That study has reported an excess weight gain of 3 - $6 \mathrm{~kg}$ with one or more 'short' pregnancies. In the present study, no additional waist circumference and WHtR increase associated with short pregnancies supports the theory that increased girth associated with birth(s) may be partially related to changes in maternal physical structure occurring relatively late in gestation as well as increased central adiposity during early gestation.

The negative association of short pregnancy with obesity markers further suggests that pregnancy is not an accurate indicator of reproductive stress. Parity does not provide information about miscarriages, abortions, or stillbirths, and the spacing of pregnancies as well as the length of breastfeeding are not taken into account. Also, if women of poor health are unable to bear more children, high-parity women may include only the healthiest individuals [31].

Limitations of our study include lack of information on changes in dietary intake and other lifestyle factors associated with parenting, and determinants of reproductive patterns. This study is further limited by the crosssectional design, which did not permit inferences about the causal direction of the relationship or clear identification of age and cohort effects. This study also cannot differentiate whether the relationships found were from child bearing or child rearing [6]. Another limitation of this study was the absence of primiparous women as this study was originally designed to investigate parent-child relation for obesity.

Despite these limitations, the strengths of our study are its community-based design and its large, diverse sample of young women of childbearing age. The large sample of a particular caste women strengthened the race/ethnicity-specific analyses. Further, our findings are consistent with previous studies showing an association of high BMI with greater pregnancy-related weight gains. Our findings also support the conclusion that maternal body size is an effect modifier in the association of one or more short pregnancies.

\section{CONCLUSION}

Our findings demonstrate that childbirth increases the risk of becoming obese. Based on these findings, obesity-prevention efforts should target women during the postpartum period. One recommendation would be to identify the women at greatest risk-normal-weight women who become overweight, overweight women, and women who are obese at the 6-week postpartum visit - and encourage them to lose weight, giving them the target of returning to their pre-pregnancy weight by 1 year postpartum. Until more clinical and community-based weightmanagement interventions are developed, these women should be given access to extant weight-management resources, and their weight should be closely monitored within the first year postpartum.

\section{REFERENCES}

[1] Heliovaara, H., and Aromaa, A. (1981) Parity and obesity. Journal of Epidemiology \& Community Health, 35, 197199. doi:10.1136/jech.35.3.197

[2] Den Tonkelaar, I., et al. (1990) Fat distribution in relation to age, degree of obesity, smoking habits, parity and estrogen use: A cross-sectional study in 11825 Dutch women participating in the DOM-project. International Journal of Obesity and Related Metabolic Disorders, 14, 753761.

[3] Arroyo, P., et al. (1995) Parity and the prevalence of overweight. International Journal of Gynaecology \& Obstetrics, 48, 269-272. doi:10.1016/0020-7292(94)02284-6

[4] Coitinho, D.C., Sichieri, R. and D'Aquino Benicio, M.H. (2001) Obesity and weight change related to parity and breastfeeding among parous women in Brazil. Public Health Nutrition, 4, 865-870. doi:10.1079/PHN2001125

[5] Smith, D.E., et al. (1994) Longitudinal changes in adiposity associated with pregnancy: The CARDIA study. The Journal of the American Medical Association, 271, 1747-1790. doi:10.1001/jama.1994.03510460039030

[6] Williamson, D.F., et al. (1994) A prospective study of childbearing and 10-year weight gain in US white women 25 to 45 years of age. International Journal of Obesity and Related Metabolic Disorders, 18, 561-569.

[7] Erem, C., et al. (2004) Prevalance of obesity and associated factors in a Turkish population (Trabzon city, Turkey). Obesity Research, 12, 1117-1127.

doi:10.1038/oby.2004.140

[8] Bastian, L.A., et al. (2005) Cache county study on memory, health, and aging. Number of children and the risk of obesity in older women. Preventive Medicine, 40, 99-104. doi:10.1016/j.ypmed.2004.05.007

[9] Gunderson, E.P. and Abrams, B. (2000) Epidemiology of gestational weight gain and body weight changes after pregnancy. Epidemiologic Reviews, 22, 261-274. doi:10.1093/oxfordjournals.epirev.a018038

[10] Gunderson, E.P., Abrams, B. and Selvin, S. (2000) The relative importance of gestational gain and maternal characteristics associated with the risk of becoming overweight after pregnancy. International Journal of Obesity and Related Metabolic Disorders, 24, 1660-1668. doi:10.1038/sj.ijo.0801456

[11] Clapp, J.F., et al. (1988) Maternal physiologic adaptations to early human pregnancy. American Journal of $\mathrm{Ob}$ stetrics \& Gynecology, 159, 1456-1460. 
[12] Gunderson, E.P., et al. (2004) Excess gains in weight and waist circumference associated with childbearing: The coronary artery risk development in young adults study (CARDIA). International Journal of Obesity and Related Metabolic Disorders, 28, 525-535. doi:10.1038/sj.ijo.0802551

[13] Gupta, S. and Kapoor, S. (2010) Sex differences in blood pressure levels and its association with obesity indices: Who is at greater risk. Ethnicity Disease, 20, 370-375.

[14] Hayes, D. and Ross, C.E. (1987) Concern with appearance, health beliefs, and eating habits. Journal of Health and Socical Behavior, 28, 120-30. doi:10.2307/2137126

[15] Sobal, J. (2004) Sociological analysis of the stigmatization of obesity. In: Germov, J. and Williams, L., Eds., $A$ Sociology of Food and Nutrition: The Social Appetite. 2nd Edition, Oxford University Press, Melbourne.

[16] World Health Organization. (1998) Obesity-Preventing and managing the global epidemic: Report of a WHO consultation on obesity. World Health Organization, Geneva.

[17] Alberti, K.G.M.M., et al. (2009) Harmonizing the metabolic syndrome: A joint interim statement of the international diabetes federation task force on epidemiology and prevention; National Heart, Lung, and Blood Institute; American Heart Association; World Heart Federation; International Atherosclerosis Society; and International Association for the Study of Obesity. Circulation, 120, 16401645. doi:10.1161/CIRCULATIONAHA.109.192644

[18] Ashwell, M., Cole, T.J. and Dixon, A.K. (1985) Obesity: New insight into the anthropometric classification of fat distribution shown by computed tomography. British Medical Journal, 290, 1692-1694. doi:10.1136/bmj.290.6483.1692

[19] Rossner, S. (1992) Short communication: Pregnancy, weight cycling and weight gain in obesity. International Journal of Obesity and Related Metabolic Disorders, 16, 145-147.

[20] Kaye, S.A., et al. (1990) The association of body fat distribution with lifestyle and reproductive factors in a population study of postmenopausal women. International Journal of Obesity and Related Metabolic Disorders, 14, 583591.

[21] Kapoor, S., Verma, S. and Bhutani, M. (1997) Structural changes during pregnancy and lactation among first parity women. Journal of Human Ecology, 8, 241-248.

[22] Greene, G.W., et al. (1988) Postpartum weight change: How much of the weight gained in pregnancy will be lost after delivery? Obstetric \& Gynecology, 71, 701-707.

[23] Ohlin, A. and Rossner, S. (1994) Trends in eating patterns, physical activity and socio-demographic factors in relation to postpartum body weight development. British Journal of Nutrition, 71, 457-470. doi:10.1079/BJN19940155

[24] Harris, H.E., Ellison, G.T., Clement, S., et al. (1999) Relative importance of heritable characteristics and lifestyle in the development of maternal obesity. Journal of Epidemiology \& Community Health, 53, 66-74. doi:10.1136/jech.53.2.66

[25] Sohlstrom, A. and Forsum, E. (1995). Changes in adipose tissue volume and distribution during reproduction in Swedish women as assessed by magnetic resonance imaging. The American Journal of Clinical Nutrition, 61, 287-295.

[26] Soltani, H. and Fraser, R.B. (2000) A longitudinal study of maternal anthropometric changes in normal weight, overweight, and obese women during pregnancy and postpartum. British Journal of Nutrition, 84, 95-101. doi:10.1017/S0007114500001276

[27] Weng, H.H., et al. (2004) Number of children associated with obesity in middle aged women and men: Results from the health and retirement study. Journal of Woman Health, 13, 99-117.

[28] Troisi, R.J., et al. (1995) Relation of body fat distribution to reproductive factors in pre- and postmenopausal women. Obesity Research, 3, 143-151.

[29] Wolfe, W.S., et al. (1997) Parity associated body weight: Modification by socio-demographic and behavioral factors. Obesity Research, 5, 131-141.

[30] Rosenberg, L., et al. (2003) A prospective study of the effect of childbearing on weight gain in African-American women. Obesity Research, 11, 1526-1535. doi:10.1038/oby.2003.204

[31] Winicoff, B. (1983) The effects of birth spacing on child and maternal health. Studies in Family Planning, 14, 231245. doi:10.2307/1965748 